\title{
Prevalence of gonococcal conjunctivitis in adults and neonates
}

L McAnena', SJ Knowles², A Curry ${ }^{3}$ and

L Cassidy ${ }^{1}$

\begin{abstract}
Purpose To report the prevalence of gonococcal conjunctivitis (GC) presenting to a tertiary referral maternity hospital (NMH) and a tertiary referral ophthalmic hospital (RVEEH) from 2011 to 2013 and describe the demographics, clinical presentation, and antibiotic susceptibility of Neisseria gonorrhoeae ocular infections.

Methods Demographic, clinical, and microbiological data were collected from patients with laboratory confirmed GC. Results There were 27556 live births at NMH during the study period, and no case of neonatal GC was identified. Fourteen cases of GC were identified at RVEEH in this period, representing a prevalence of 0.19 cases per $\mathbf{1 0 0 0}$ eye emergency attendees. Antibiotic susceptibility data were available on nine cases, of which, all were ceftriaxoneand ciprofloxacin sensitive. $64.3 \%$ of patients were male, with a mean age of 18 years. The mean duration of symptoms was 3 days. All patients presented with unilateral conjunctival injection and purulent discharge. Eight cases had visual impairment at presentation and their mean visual acuity was 6/15. Corneal involvement was present in $25 \%$ of patients. Uveitis was not detected. On receipt of positive culture and/or PCR results, treatment was altered in two thirds of patients. All patients were referred for

\section{Introduction}

Traditionally, gonococcal conjunctivitis (GC) was considered rare in adults and was predominantly a disease of neonates. ${ }^{1}$ In recent years, the incidence of gonorrhoeal infection has increased sevenfold from 6.9 per 100000 in 2003 to 49.2 per 100000 in 2012 in the Eastern counties of Ireland. ${ }^{2}$ Similar increases have been seen in England, where the incidence increased by 66 percent from 2010 to $2012^{3,4}$ and in Australia, where the rate of gonorrhoea increased from 35.8 per 100000 in 2005 to 60.0 per 100000 in 2012. ${ }^{5}$

GC typically presents with a severe mucopurulent conjunctivitis with associated lid oedema, tenderness and often preauricular lymphadenopathy. GC may be complicated by uveitis or severe keratitis, sometimes leading to corneal perforation necessitating corneal graft repair, so prompt diagnosis and treatment are essential. $^{6-9}$

There has been an increase in antibiotic resistance amongst gonococcal strains in recent years, including resistance to penicillin, fluoroquinolones, and more recently, oral cephalosporins. ${ }^{10-12}$ Increasing antimicrobial resistance internationally has sparked concerns that some Neisseria gonorrhoeae strains may become pan-resistant in the near future.
\end{abstract} full STI screening and all patients showed a full clinical recovery 1 week posttreatment. Conclusion We observed that GC presented in young adults with a male predominance and was rare in neonates. In cases of unilateral purulent conjunctivitis, there should be a high clinical suspicion of GC, early swab for PCR and culture, and knowledge of current CDC-recommended antibiotic guidelines.

Eye (2015) 29, 875-880; doi:10.1038/eye.2015.57; published online 24 April 2015

\section{The aims of this study}

To investigate the prevalence of GC at two specialist Dublin hospitals: the Royal Victoria Eye and Ear Hospital (RVEEH) and the National Maternity Hospital (NMH) from 2011 to 2013

To examine antibiotic susceptibility of the N. gonorrhoeae isolates.

To examine the demographics and patterns of clinical presentation and outcome. inclusive.
${ }^{1}$ Department of Ophthalmology, Royal Victoria Eye and Ear Hospital, Dublin, Ireland

${ }^{2}$ Department of Microbiology, Royal Victoria Eye and Ear Hospital, National Maternity Hospital, Dublin, Ireland

${ }^{3}$ Department of Microbiology, National Maternity Hospital, Holles St, Dublin, Ireland

Correspondence: L McAnena, Royal Victoria Eye and Ear Hospital, Adelaide Road, Dublin 2, Ireland

Tel: +353 1 6644600;

Fax: +35316761858.

E-mail: Imcanena@yahoo. co.uk

Received: 13 June 2014 Accepted in revised form: 1 February 2015 Published online: 24 April 2015 


\section{Materials and methods}

\section{Study population and setting}

This study was conducted in two tertiary referral specialist hospitals-RVEEH a specialist Ophthalmic and Ear, Nose, and Throat hospital and NMH a large maternity hospital, both in Dublin, Ireland. During the study period $12.77 \%$ of all infants born in the Republic of Ireland were delivered at the NMH. The microbiology laboratory at the NMH serves both hospitals.

\section{Data collection and definitions}

The laboratory information system was searched for all bacterial conjunctival swabs and all ocular gonococcal PCR swabs obtained from any patient attending the RVEEH and any newborn attending $\mathrm{NMH}$ between 1 January 2011 and 31 December 2013 inclusive. A confirmed diagnosis of GC was defined as either a positive culture or PCR test. A retrospective analysis of the medical records of each confirmed case was conducted focusing on patient demographics, clinical presentation, treatment, and clinical outcomes.

\section{Laboratory methods}

Bacterial Culture: all eye swabs (NMH and RVEEH) are inoculated onto Columbia blood agar and chocolate agar and streaked out for individual colonies. Agar plates are incubated at $35^{\circ} \mathrm{C}$ in $\mathrm{CO}_{2}$ for $40-48 \mathrm{~h}$. A direct Gram stain from the swab is performed on all neonatal samples and if requested from all other patients. Following incubation, agar plates are examined for potential pathogens on a daily basis. Potential N. gonorrhoeae are confirmed with Gram stain and are identified using API NH (BioMerieux) and Rapid NHID (Fannins) methods.

Susceptibility testing is referred externally to a reference laboratory. The antibiotic disc diffusion method adheres to the Clinical and Laboratory Standards Institute (CLSI) method and uses GC-S agar for susceptibility testing. Antibiotics tested are ceftriaxone, ciprofloxacin, penicillin, spectinomycin, and tetracycline.

Nucleic Acid Amplification Testing: a multiplex PCR is performed for both Chlamydia trachomatis and $N$. gonorrhoeae in the National Virus Reference Laboratory. An APTIMA swab is used for sample collection and a qualitative PCR using the commercial kit APTIMA (Gen Probe Inc.) is performed.

\section{Statistical analysis}

Frequencies and proportions were used to describe the data. This was carried out using Epilnfo, Centers for Disease Control and Prevention, Atlanta, GA, (http://wwwn.cdc.gov/epiinfo/). A $P$-value $<0.05$ is considered statistically significant.

This study has received institutional approval from the Audit Committee at the Royal Victoria Eye and Ear Hospital, Dublin, and meets the tenets of the Declaration of Helsinki.

\section{Results}

\section{Prevalence}

There were a total of 74814 presentations for all eye problems to the Ophthalmic Accident and Emergency Department (OAED) at RVEEH during the three year study period. Bacterial conjunctival swabs were obtained from 3088 patients and a further 791 swabs were obtained for N. gonorrhoeae PCR. Fourteen cases of GC were identified at RVEEH, all presenting to the OAED during the study period.

There were 27556 births at the NMH during the study period. Bacterial conjunctival swabs were obtained from 676 newborns and 53 PCR swabs were obtained for $N$. gonorrhoeae. No case of GC was identified in a newborn at the $\mathrm{NMH}$ during the 3-year study period.

The prevalence of GC was 0.19 cases per 1000 eye emergency attendees (95\% confidence interval: 0.09-0.29 cases). GC was more common among attendees at an Eye Emergency Department (14/74814) than neonates born at a Maternity Hospital $(0 / 27556 ; P=0.02)$.

The laboratory method used to diagnose GC is identified in Table 1 . The sensitivity of the culture method was $80 \%(8 / 10)$ and the sensitivity of the PCR method was $90 \%$ (9/10), when both methods were performed. In both cases where there was a false-negative culture result and a positive PCR result, there was a delay of 2-3 days in receipt of the swab at the laboratory for culture.

\section{Antibiotic resistance}

Antibiotic susceptibility data were available for nine patients. All isolates were susceptible to ceftriaxone (nine tested), ciprofloxacin (eight tested) and spectinomycin (six tested). The majority of isolates were penicillin

Table 1 Laboratory method for identification of Neisseria gonorrhoeae $(n=14)$

\begin{tabular}{lccc}
\hline & $\begin{array}{c}\text { PCR } \\
\text { positive }\end{array}$ & $\begin{array}{c}\text { PCR } \\
\text { invalid }\end{array}$ & $\begin{array}{c}\text { PCR not } \\
\text { performed }\end{array}$ \\
\hline $\begin{array}{l}\text { Culture positive } \\
\text { Culture negative }\end{array}$ & 7 & 1 & 3 \\
$\begin{array}{l}\text { Culture not } \\
\text { performed }\end{array}$ & 1 & - & - \\
\hline
\end{tabular}


nonsusceptible (six of eight isolates tested) and tetracycline nonsusceptible (four of five isolates tested).

\section{Gonococcal cases}

$64.3 \%$ of patients were male, with an average age of 18 years (range 3-24 years). Medical records from 12 patients were available for review. The mean duration of symptoms at presentation was 3 days (range 1-7 days). All patients presented with unilateral conjunctival injection and purulent discharge (see Table 2).

Eight cases had visual impairment at presentation and their mean visual acuity was 6/15. Corneal involvement was present in $25 \%$ of patients and was exclusively mild, in the form of superficial punctate erosions (SPE's).

Uveitis or corneal perforation was not detected.

Initial treatment regimens varied before receipt of positive PCR or culture. Four patients (33.3\%) received topical antibiotics only (for bacterial conjunctivitis), whereas 3 patients (25\%) received empiric treatment targeted at chlamydial/gonococcal conjunctivitis. Five patients $(41.7 \%)$ were initially treated for presumed preseptal cellulitis, two of whom were admitted to hospital.

The average time for receipt of swab results was 4.4 days. On receipt of the positive culture and/or PCR results, treatment was changed in eight patients $(66.7 \%)$ to target gonococcal infection. One case was a three-year-old child, who was referred to a children's hospital for further treatment and follow-up. See Table 3 for details of treatment administered before, and after positive swab results were received.

Ten of the 11 adult cases attended for follow-up within 1 week of completion of treatment of GC and all cases showed full resolution of clinical signs by this time. All patients were referred for sexually transmitted infection (STI) work-up at a seperate centre. Of the 11 adults referred for STI screening, two refused to attend, as they strongly denied any sexual intercourse. One of these patients was a 15-year-old male, the other a 17-year-old female. Of the nine patients who did get STI screening, follow-up is known on only three, as the remainder with their ocular symptoms resolved, did not follow-up at our eye hospital. Of these three patients, the STI screen was negative in two, and one tested positive for pharyngeal gonorrhoea.

\section{Discussion}

In our experience in recent years, gonococcal conjunctivitis has presented predominantly in teenagers and young adults, with a 1.8:1 male: female gender distribution. This likely reflects the increasing rates of gonococcal infection in Ireland over the past 10 years.
Table 2 Clinical presentation $(n=12)$

\begin{tabular}{lc}
\hline Sign & Prevalence \\
\hline Injection & $12(100 \%)$ \\
Purulent discharge & $12(100 \%)$ \\
Lid swelling & $8(66.6 \%)$ \\
Chemosis & $6(50 \%)$ \\
Corneal involvement (SPE's only) & $3(25 \%)$ \\
Uveitis & $0(0 \%)$ \\
\hline
\end{tabular}

Despite the increase in cases of gonorrhoea detected nationally, GC was very rare among newborns at our hospital, which delivered one in eight of infants born in the Republic of Ireland during the study period.

No topical prophylaxis was used in the maternity hospital for ophthalmia neonatorum. A similar study performed at Cork University Hospital reported two adult and only one neonatal case of GC from July 2002 to December 2006. ${ }^{13}$

We hypothesise that the disparity between the rising incidence of gonorrhoeal infection amongst adults and the rarity of GC in neonates may be explained by the difference between the demographics of the gonorrheal presentations and the mean maternal age in Ireland. The mean maternal age in Ireland in 2011 and 2012 was 31.85 years. ${ }^{14}$ In contrast, the recent gonorrhoea outbreak has been predominantly amongst men who have sex with men (MSM) and young heterosexual populations as outlined in a recent report of incidence patterns of gonorrhoea in Ireland in the first quarter of 2013 by the Health Protection and Surveillance Centre (HPSC) of Ireland. ${ }^{15}$ This reports a median age of 26 years, a male : female ratio of $5.3: 1$ and $56 \%$ of all cases occurring in MSM. In this same report, only 2 of the total of 223 reported cases of gonorrhoea in this period were seen in heterosexual females age 30-34 years. Of course, we can only draw inference from these figures and further research may be required to explain this disparity.

PCR is now considered the gold standard for diagnosing gonococcal infection. ${ }^{16}$ Culture is less sensitive as the organism is labile and requires rapid transport to the laboratory from the bedside in order to successfully grow the organism under normal laboratory conditions. If gonococcal infection is not clinically suspected and only a conjunctivial culture swab is obtained, it is possible that cases may be missed if there is a delay in transport to the laboratory for processing.

In our case series, the clinical course was relatively mild. Lid involvement was common, and may resemble preseptal cellulitis. ${ }^{17}$

It is notable that empiric treatment targeted at gonorrhoeal or chlamydial conjunctivits was administered to just one quarter of patients at presentation and these antibiotic regimens were variable. 


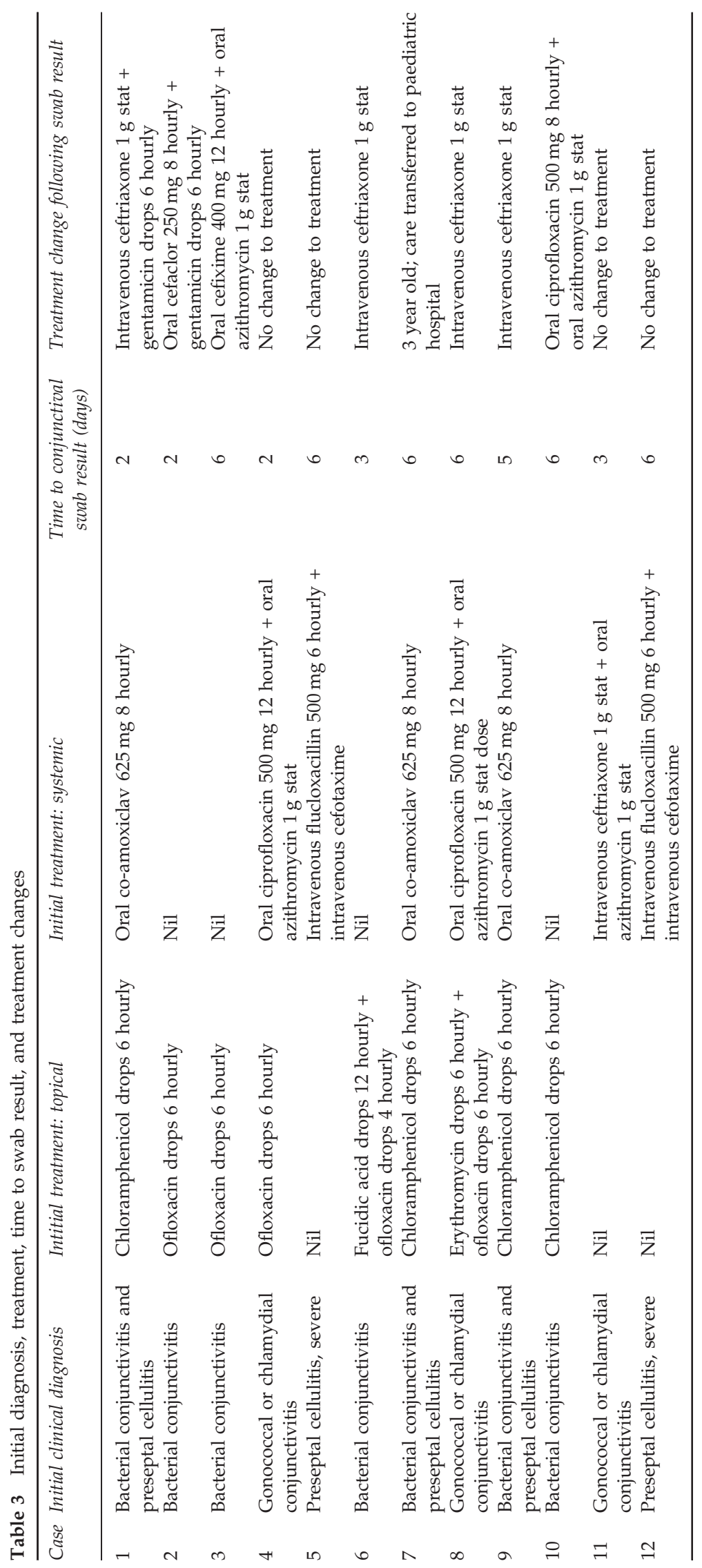


The majority of cases were initially treated for bacterial conjunctivitis and/or preseptal cellulitis before receipt of a positive swab result. This is perhaps indicative of the relatively low clinical suspicion of GC and subsequent potential for misdiagnosis. All cases subsequently received either a cephalosporin and/or azithromycin treatment with subsequent full clinical resolution of their infection. Treatment regemins varied greatly, however, highlighting the need for better knowledge of antibiotic guidelines amongst practitioners. This is especially important in the current era of increased antibiotic resistance.

The majority of cases seen were penicillin and tetracycline nonsusceptible. No antibiotic resistance to ceftriaxone, ciprofloxacin, and spectinomycin was detected, although the number of isolates tested was small. In light of the recent rise in antibiotic resistance amongst gonococcal strains, the current CDCrecommended treatment regime for gonococcal conjunctivitis is ceftriaxone $1 \mathrm{~g}$, intramuscularly. ${ }^{10}$ Adjuvant therapy can include saline lavage and topical chloramphenicol or ofloxacin. ${ }^{18,19}$ Patients should also be treated presumptively for concurrent Chlamydia trachomatis infection with either azithromycin $1 \mathrm{~g}$ orally as a single dose or doxycycline $100 \mathrm{mg}$ orally twice daily for 7 days. $^{10}$

Among the cases identified, two teenagers strongly denied sexual intercourse and we hypothesize that they may have acquired the infection via hand transmission.

\section{Study limitations}

This study is retrospective and not all denominators had evidence of conjunctivitis or ocular infection. We examined the proportion of GC cases amongst all presentations to an OAED at a single centre (RVEEH) and therefore, a true incidence of adult GC cannot be deduced. In contrast, the neonatal sample represents the proportion of GC diagnoses amongst all neonates at one centre. Another limitation of the study is that we may be underestimating the prevalence of this infection, especially in the RVEEH, as the microbiology laboratory is off-site and there may be a delay in inoculation of the bacterial swab in the laboratory and possible falsenegative culture results, especially if PCR is not performed.

\section{Conclusion}

A diagnosis of GC should be suspected in young adults presenting with unilateral purulent conjunctivitis and/or preseptal cellulitis of short duration. We recommend PCR testing if there is a clinical suspicion of GC and prompt culture of conjunctival swabs. Ophthalmologists should be aware of current antibiotic guidance for treatment of GC. All cases should be referred to a specialist STI clinic for further screening and contact tracing.

\section{Summary}

What was known before

- Gonococcal conjunctivitis (GC) is considered a disease of neonates and rare in adults.

What this study adds

- This study suggests that, in recent years, GC is presenting more commonly in young adults, with a male predominance, reflecting a current epidemic of gonorrheal infection in Ireland.

- The prevalence of neonatal GC is, in contrast, very low.

- It also highlights the importance of performing swab for culture and PCR, in light of rising antibiotic resistance and new antibiotic-prescribing guidelines.

\section{Conflict of interest}

The authors declare no conflict of interest.

\section{References}

1 Lee JS, Choi HY, Lee JE, Lee SH, Oum BS. Gonococcal keratoconjunctivitis in adults. Eye 2002; 16(5): 646-649.

2 Fitzgerald M, Cooney F, Ennis O, Downes P, Clarke S. Gonorrhoea - a major public health challenge in Dublin, Wicklow and Kildare. Epi-Insight 2013; 14(2): http://ndsc.newsweaver.ie/epiinsight/16xjz7b64shqldxs0g4hal? $a=1 \& p=31757965 \& t=17517774$.

3 Public Health England. Table 8: Number \& rates of gonorrhoea diagnoses in England, 2003 - 2012. Available from http:/ /www.hpa.org.uk/webc/HPAwebFile/ HPAweb_C/1281953082152.

4 Savage EJ, Marsh K, Duffell S, Ison CA, Zaman A, Hughes G. Rapid increase in gonorrhoea and syphilis diagnoses in England in 2011. Euro Surveill 2012; 17(29): pii: 20224.

5 Lahra M. Australian Gonococcal Surveillance Programme annual report, 2012. Commun Dis Intell $Q$ Rep 2013; 37(3): E86-E87.

6 Ullman S, Roussel TJ, Culbertson WW, Forster RK, Alfonso $\mathrm{E}$, Mendelsohn AD et al. Neisseria gonorrhoeae keratoconjunctivitis. Ophthalmology 1987; 94(5): 525-531.

7 Ullman S, Roussel TJ, Forster RK. Gonococcal keratoconjunctivitis. Surv Ophthalmol 1987; 32(3): 199-208.

8 Wan WL, Farkas GC, May WN, Robin JB. The clinical characteristics and course of adult gonococcal conjunctivitis. Am J Ophthalmol 1986; 102(5): 575-583.

9 Kawashima M, Kawakita T, Den S, Tomita M, Shimazaki J. Surgical management of corneal perforation secondary to gonococcal keratoconjunctivitis. Eye (Lond) 2009; 23(2): 339-344.

10 Centers for Disease Control and Prevention (CDC) Update to CDC's Sexually transmitted diseases treatment guidelines, 2010: oral cephalosporins no longer a recommended treatment for gonococcal infections. MMWR Morb Mortal Wkly Rep 2012; 61(31): 590-594. 
11 Hopkins S, Coleman C, Kelleher M, Keating S, Clarke S, O'Connell B et al. Increasing resistance to ciprofloxacin among isolates of Neisseria gonorrhoeae in Dublin. Ir Med J 2005; 98(7): 208-209.

12 Unemo M, Golparian D, Potocnik M, Jeverica S. Treatment failure of pharyngeal gonorrhoea with internationally recommended first-line ceftriaxone verified in Slovenia, September 2011. Euro Surveill 2012; 17(25): pii: 20200.

13 Quirke M, Cullinane A. Recent trends in chlamydial and gonococcal conjunctivitis among neonates and adults in an Irish hospital. Int J Infect Dis 2008; 12(4): 371-373.

14 Central Statistics Office. Average Age of Mother Classified by Marital Status 2013. Available from http:/ / www.cso.ie/ en/statistics/birthsdeathsandmarriages/ averageageofmotherclassifiedbymaritalstatus/.
15 Fizgerald M, Igoe D, Cooney F. Control group use surveillance findings to target response to gonorrhoea increase. Epi-Insight 2013; 14: 10.

16 Papp JR, Schachter J, Gaydos CA, Van Der Pol B. Recommendations for the Laboratory-Based Detection of Chlamydia trachomatis and Neisseria gonorrhoeae-2014. MMWR Recomm Rep 2014; 63(no RR-2): 1-19.

17 Hegde V, Smith G, Choi J, Pagliarini S. A case of gonococcal kerato-conjunctivitis mimicking orbital cellulitis. Acta Ophthalmol Scand 2005; 83(4): 511-512.

18 Jackson TL. Moorfields Manual of Ophthalmology. Mosby, Elsevier: Amsterdam, Netherlands, 2008.

19 Gerstenblith AT, Rabinowitz MP. The Wills Eye Manual. 6th edn. Lippincott Williams \& Wilkins: Philadelphia, 2012. 